\title{
PHYSICS OF THE QUARK-GLUON PLASMA
}

\author{
Janos Polonyi \\ Laboratory of Theoretical Physics \\ Louis Pasteur University \\ 3 rue de l'Université 67087 Strasbourg Cedex France \\ CRN, CNRS-IN2P3 \\ 23 rue du Loess 67200 Strasbourg Cedex France \\ Department of Atomic Physics \\ L. Eötvös University \\ Puskin u. 5-7 1088 Budapest Hungary
}

\begin{abstract}
Some features of the high temperature gluonic matter, such as the breakdown of the fundamental group symmetry by the kinetic energy, the screening of test quarks by some unusual gluon states and the explanation of the absence of isolated quarks in the vacuum without the help of infinities are presented in this talk. Special attention is paid to separate the dynamical input inferred from the numerical results of lattice gauge theory from the kinematics.
\end{abstract}

\section{INTRODUCTION}

Contemporary physics progresses towards opposite ends. On the one hand, one presses for the discovering the fundamental laws in their ultimate simplicity. This is the realm of subatomic physics where the elementary building blocks of matter are studied. On the other hand, the description of complex systems which are made up by simple elements and rules is sought. Ergodicity or its absence, collective phenomena and statistical physics label this direction. The topic of the relativistic heavy ion collisions, RHIC, represents a meeting ground for these two directions inasmuch as it involves the interactions of elementary particles, quarks, and the collective phenomena, phase transition and fragmentation. It is not sure that we shall ultimately arrive at the understanding of the remnants of an energetic heavy ion collision but we certainly have to use a vast area of physics and a good deal of intuition in the analysis. The attempt at synthesis is specially useful in our situation where the furthering of our experimental inquiry in the microscopic domain is severely limited by economical considerations.

The interest of the community was increased considerably in RHIC after realizing that a "new phase of matter" might be at stakes at this experiment. It is difficult sometime not to be lost in the rich details of this problem but we should remember that this experiment is worth for the concentration of our resources only if it produces more than a quantitative change in particle production, when it helps to discover qualitatively new laws of matter. RHIC can add 
news compared to the $p p$ collisions if the collective modes become important in modifying the medium where the elementary interactions take place.

I do not attempt to review the theoretical aspects of the strong interactions from the point of view of the RHIC. Instead I shall be contented to present some thoughts about quark confinement what I find interesting in this subject. The confinement of quarks represents several problems in itself because there is no other interaction which can shield the elementary constituents of a composite state as effectively then the confining forces between quarks. The understanding of this mechanism would remove one of the most troublesome obstacle of particle physics. After having identified the confining mechanism it is equally challenging to understand its absence when the matter is under extreme environments. It turned out that the deconfining phase transition is fundamentally different from other phase transitions. A brief sketch of this latter claim is the main subject of this talk.

\section{CONFINEMENT MECHANISMS}

One can distinguish soft and hard confinement mechanisms. The former stands for the screening of a quark [1] when we try to separate it from a hadron. This screening is achieved by the creation of a string of mesons. This phenomenon seems similar to the supercritical state of the QED vacuum [2]. Such a screening of the isolated quarks is called soft because it involves low energies. In order to have this mechanism operative for hadrons where $Z=2,3$ one needs strong coupling constant. This is provided by the anti-screening of the asymptotically free gluon exchanges. The hard confinement mechanism is what prevents the separation of static test quarks even in the absence of the quark Dirac sea in the pure gluonic theory. This is believed to happen by the flux-tube formation.

It is very difficult to separate the soft and the hard confinement mechanisms in hadronic reactions. The real flux-tube is broken by the soft confining mechanism and is only seen indirectly in the Regge trajectories and the heavy meson spectroscopy. In lacking of experimental guidance we have to look for theoretical starting points. It seems reasonable that the description of the pure gluonic vacuum is a prerequisite for the understanding of the soft confinement mechanism. Furthermore both the generation of the strong coupling strength and the hard confinement mechanism seem to arise from the non-perturbative long-range modes of the pure gluonic vacuum. Only the hard confinement mechanism is addressed here and the quark-anti quark vacuum polarization which is responsible for the soft mechanism will be neglected. The qualitative arguments presented below remain valid in their presence so long as the proper ensemble is used [3].

The best evidence for the hard confinement mechanism comes from the numerical studies of lattice QCD in the quenched approximation, i.e. without dynamical quarks. As far as the origin of the string tension is concerned the dual superconductor analogies, [4], and the haaron-gas description, [5], seem to provide a consistent framework to describe the numerical experiences. They are based on rather different physical scenarios: While the superconductor picture uses the dual Meissner effect the haaron-gas description refers to localization. It remains to seen if these mechanisms which are supported by the numerical results of lattice QCD can be brought to a common ground.

\section{DECONFINEMENT PHASE TRANSITIONS}

Instead of pursuing the issue of the confinement mechanisms we turn to the question of deconfinement. Our starting point will be the deconfining phase transition because its careful study should provides us the clue of confinement and deconfinement in the same time. 
The studies of QCD at high temperature became interesting because the smallness of the running coupling constant at high temperature suggested that the theory becomes perturbative and reveals its elementary constituents. But the smallness of the effective coupling strength at the average kinetic energy is not enough to make the system perturbative. Infrared divergences continue to plague the perturbation expansion at arbitrary high temperature. The other expectation namely that QCD looses the confining forces, [6], found out to be correct.

The appearance of new degrees of freedom can be expected from the quantum field theory model where the hadrons are treated as pointlike particles. Such non-gauged hadron models are non-asymtotically free and their only renormalizable version is expected to describe noninteracting hadrons. The non-renormalizability of the interactive effective theory implies that the model remains useful only up to a certain finite value of the UV cut-off. Thus new physics must come beyond this maximal cut-off scale. The new degrees of freedom, supposedly the quarks and gluons can be made more explicit by reaching the maximal cut-off scale not only in the energy of an isolated reaction but instead in a large region in space for an extended time. One expects to trigger a phase transition in this manner. One can pump energy into a system (i) thermally, (ii) chemically or (iii) mechanically. Either of these methods can be used to create the new phase.

(i) The finite temperature phase transition in hadronic matter was predicted first by Hagedorn who noted that the level density of the observed hadronic resonances seems to follow an exponential increase with the energy, $n_{h}(E) \approx e^{E / T_{H}}$. Thus the simple one-particle approximation for the hadronic partition function,

$$
Z=\int_{0}^{\infty} d E n_{h}(E) e^{-\frac{E}{T}}=\int_{0}^{\infty} d E e^{-E\left(\frac{1}{T_{H}}-\frac{1}{T}\right)}
$$

diverges for $T>T_{H}$. One has to provide infinite amount of energy to raise the temperature beyond this limiting value.

The strong coupling expansion in hamiltonian lattice gauge theory gives similar result. Consider $\mathrm{U}(1)$ gauge theory for simplicity where the eigenstates of the strong coupling hamiltonian which includes the kinetic energy only,

$$
H=-\frac{1}{2} \sum_{x, i} \frac{\delta^{2}}{\delta A_{i}^{2}(\mathbf{x})}
$$

are

$$
\Psi_{\ell_{i}(\mathbf{x})}\left[A_{i}(\mathbf{x})\right]=e^{i g \sum_{\mathbf{x}, i} \ell_{i}(\mathbf{x}) A_{i}(\mathbf{x})},
$$

where $\ell_{i}(\mathbf{x})$ is an integer valued vector field which counts the electric excitations along the links. Let us approximate the partition function of a static $e^{+} e^{-}$pair by taking into account the states where the links are at most once excited. The excitations form a string connecting the location of the two charges due to Gauss' law. Thus the partition function of the gauge field in the $e^{+} e^{-}$ sector is

$$
Z=\sum_{\Gamma} e^{-\frac{g^{2}}{2} L[\Gamma]}
$$

where the summation is over all strings, $\Gamma$, with given end points and $L$ stands for the length of the strings. The number of strings for large $L$ is given by $n_{s}(L) \approx 5^{c L}$ where $c=O\left(L^{0}\right)$ since 
each additional link to the string can be chosen from one of five different directions. Thus we have

$$
Z=\sum_{L=0}^{\infty} e^{-L\left(\frac{g^{2}}{2 T}-c \ln 5\right)} .
$$

The similarity with Hagedorn's scenario is obvious with the only difference that it is the entropy of the gluons rather than the hadrons which makes the partition function divergent. The strong coupling picture allows us to see what changes as the temperature is increased beyond the critical

temperature, $T_{d e c}=\frac{g^{2}}{2 c \ln 5}$. For $T<<T_{d e c}$ the partition function is dominated by the straight string connecting the sources and the string tension is $\sigma=g^{2} / 2$. When $T>>T_{d e c}$ then they are the long strings wandering around in space which are important and the force between the charges is vanishing for large separation.

The existence of a phase transition where the string tension vanishes beyond $T_{d e c}$ has been confirmed by numerical studies of gauge models with compact gauge group. The compactness is essential since the boundlessness of the coordinate space of the gauge field makes the spectrum of the electric field operator discrete. The size of the coordinate space, $R=O\left(\frac{1}{g}\right)$, is proportional to the inverse of the level spacing of the canonical momentum operator, $E_{i}(\mathbf{x})=\frac{1}{i} \frac{\delta}{\delta A_{i}(\mathbf{x})}$. Thus the gap of the excitation spectrum of the strong coupling hamiltonian, (3.2), is proportional to $g^{2}$. Larger the gap, more important is the energy in the partition function (3.5) and the typical strings fluctuate in the closer vicinity of the shortest line connecting the $e^{+} e^{-}$pair.

(ii) The chemical energy pump is to squeeze hadrons into a box with high density. Imagine that hadrons as small bags containing the quarks. As the hadrons overlap the quarks may propagate freely between the bags which are in contact and we recover a quark gas in a "big" bag. This is a percolation type phase transition since the formation of a large connected region made of the individual hadrons is needed for the deconfinement. Unfortunately the quark determinant in the path integral is not positive definite for non-vansishing baryon number and the numerical methods which are based on stochastic processes are unable to confirm the presence of such phase transition.

(iii) Observe that the role of the baryon density in the previous case was to control the average hadron separation. This can be reached by changing the pressure which leads to the mechanical way of piling up energy. It is reasonable to expect that the hadrons in a gas start to overlap as the pressure is increased and similar deconfining phase transition takes place. This is equivalent with the phase transition which is supposed to be induced by the high baryon density at RHIC. The question can be raised in the thought experiment of colliding energetic ions on an anti-ion beam where hadron gas with vanishing baryon density is created. There is no problem with stochastic methods in this case. A new density matrix should be used to describe the time averages and the preliminary numerical results indicate the presence of the deconfining phase transition [7].

\section{ORDER PARAMETER OF CONFINEMENT}

The numerical studies of the gluon system indicate that the expectation value of the Polyakov line,

$$
<\operatorname{tr} \Omega(\mathbf{x})>=<P e^{\int_{0}^{\beta} d t A_{0}(\mathbf{x}, t)}>=e^{-\beta F_{q}}=\frac{Z_{\bar{q}}}{Z_{0}},
$$

is vanishing for $T<T_{d e c}$ and becomes nonzero for $T>T_{d e c}$. The first equation in (4.1) is the definition, $P$ stands for path ordering and the gauge field is antihermitean, $A_{\mu}=g A_{\mu}^{a} \frac{\lambda^{a}}{2 i}$. There are three different interpretations of $\langle\operatorname{tr} \Omega(\mathbf{x})>$ what I comment here briefly. 
1. Quark free energy: The expectation value of the Polyakov line which corresponds to the insertion of the path ordered exponential into the path integral describes the interaction of the gluons with a static external charge. Thus this expectation value gives the free energy, $F_{q}$, associated to a static quark. $Z_{\bar{q}}$ and $Z_{0}$ is the partition function for the one antiquark and the quarkless sectors. The role of $A_{0}$ in the path integration is the projection into the physical Hilbert space. One can write the partition function of the vacuum sector as

$$
\begin{aligned}
Z_{0} & =\operatorname{tr} \mathcal{P}_{0} e^{-\beta H} \\
& =\operatorname{tr} \int D[\Omega(\mathbf{x})] \hat{\Omega} e^{-\beta H} \\
& =\int D[\mathbf{A}(\mathbf{x})] \int D[\Omega(\mathbf{x})]<\mathbf{A}^{\Omega}\left|e^{-\beta H}\right| \mathbf{A}>
\end{aligned}
$$

where $\mathcal{P}_{0}$ is the projection operator into the gauge invariant sector and $\hat{\Omega}$ is the representation of the gauge transformation $\Omega(\mathbf{x})$ in the Hilbert space. The partition function in the presence of a test antiquark at the location $\mathbf{x}_{0}$ is

$$
\begin{aligned}
Z_{\bar{q}} & =\operatorname{tr} \mathcal{P}_{\bar{q}} e^{-\beta H} \\
& =\operatorname{tr} \int D[\Omega(\mathbf{x})] \Omega\left(\mathbf{x}_{0}\right) \operatorname{tr} \hat{\Omega} e^{-\beta H} \\
& =\int D[\mathbf{A}(\mathbf{x})] \int D[\Omega(\mathbf{x})]<\mathbf{A}^{\Omega}\left|e^{-\beta H}\right| \mathbf{A}>\operatorname{tr} \Omega\left(\mathbf{x}_{0}\right) .
\end{aligned}
$$

$\mathcal{P}_{\bar{q}}$ denotes the projection operator into the anti-quark sector. The non-vanishing of the expectation value (4.1) indicates that the isolated quarks are not completely suppressed.

2. Center symmetry: The center consists of global gauge transformations which commute with the gauge group. It is

$$
Z_{N}=\left\{\delta_{j k} e^{i \frac{2 \pi}{3} \ell}, \ell=1, \cdots, N\right\}
$$

for the group $S U(N)$. Gauge transformations act on the gluon and the quark fields as

$$
\begin{gathered}
A_{\mu}(x) \rightarrow A_{\mu}^{\omega}(x)=\omega(x)\left(\partial_{\mu}+A_{\mu}(x)\right) \omega^{\dagger}(x), \\
\psi(x) \rightarrow \psi^{\omega}(x)=\omega(x) \psi(x) .
\end{gathered}
$$

Global center transformations leave the gauge field invariant because they commute with the generators but change the quark field.

It is instructive to compare these relations with those for the rotational group in three space. In $S U(2)$ gauge theory the center is $Z_{2}=\{1,-1\}$ which corresponds to rotation in the color space by angle 0 or $2 \pi$. Fermions transform according to the spinor representation and they pick up the minus sign under rotation by $2 \pi$. The gauge field is in the adjoint, vector representation where the vectors remain invariant under rotation by $2 \pi$.

We write the partition function (4.2) as

$$
Z_{0}=\int D[\Omega(\mathbf{x})] e^{-\mathcal{H}[\Omega]}
$$


where $\mathcal{H}[\Omega]=-\ln \operatorname{tr} \hat{\Omega} e^{-\beta H}$. One can actually generalize this construction to any matrix element,

$$
<\mathbf{A}_{f}\left|e^{-\beta H}\right| \mathbf{A}_{i}>_{0}=\int D[\Omega(\mathbf{x})]<\mathbf{A}_{f}^{\Omega}\left|e^{-\beta H}\right| \mathbf{A}_{i}>=\int D[\Omega(\mathbf{x})] e^{-\mathcal{H}_{\mathbf{A}_{f}, \mathbf{A}_{i}}[\Omega]}
$$

with

$$
\mathcal{H}_{\mathbf{A}_{f}, \mathbf{A}_{i}}[\Omega]=-\ln <\mathbf{A}_{f}^{\Omega}\left|e^{-\beta H}\right| \mathbf{A}_{i}>\text {. }
$$

The pure gluonic amplitudes in the vacuum sector between the gluon field eigenstates $\mid \mathbf{A}_{i}>$ and $\mid \mathbf{A}_{f}>$ are center invariant in a trivial manner, $<\mathbf{A}_{f}^{z}\left|e^{-\beta H}\right| \mathbf{A}_{i}>=<\mathbf{A}_{f}\left|e^{-\beta H}\right| \mathbf{A}_{i}>$ and the pure gluonic system possesses center invariance, $\mathcal{H}[z \Omega]=\mathcal{H}[\Omega], \mathcal{H}_{\mathbf{A}_{f}, \mathbf{A}_{i}}[z \Omega]=\mathcal{H}_{\mathbf{A}_{f}, \mathbf{A}_{i}}[\Omega]$, where

$$
z=e^{i \frac{2 \pi}{3}}
$$

is a center element. The Polyakov line is a quark observable and transforms multiplicatively, $\Omega \rightarrow z \Omega$, hence it is an order parameter and the non-vanishing value of (4.1) indicates the dynamical breakdown of the center symmetry.

3. Fundamental group [8]: It is known that the wave function can be multi-valued when the coordinate space is multiply connected and this gives rise a phase representation of the fundamental group of the coordinate space. The simplest example is a particle on the circle. The trajectories on the circle fall into homotopy classes which can be characterized by the winding number. When the particle moves first along a path $\gamma_{1}$ with winding number $\nu_{1}$ after then another path, $\gamma_{2}$ with winding number $\nu_{2}$ then it performs motion along the path $\gamma_{1} \gamma_{2}$ which has winding number $\nu_{1}+\nu_{2}$. The multiplication between the homotopy classes gives the fundamental group which is $Z$ the additive group of integers for the circle. The irreducible representations of this group can be labeled by an angle $\theta$ which controls the multi-valuedness of the wave functions, $\psi(\phi+2 \pi)=e^{i \theta} \psi(\phi)$. Since the observable quantities correspond to operators which have single valued matrix elements the linear space, $\mathcal{H}_{\theta}$, consisting of the wave functions with a given $\theta$-parameter are closed with respect to the action of the physical operators and form a superselection class. The phase $\theta$ is the magnetic flux going through the circle and its appearance in observable quantities is the Aharonov-Bohm effect. One should bear in mind that the hamiltonian and some other observable are actually $\theta$ dependent.

Another example is provided by rotations in three space. Consider the wave function of an N-body system expressed by the help of the collective coordinates,

$$
\psi\left(\mathbf{x}_{1}, \cdots, \mathbf{x}_{N}\right)=e^{-i \mathbf{X P}} \mathcal{D}_{m_{1}, m_{2}}^{j}(\phi, \theta, \chi) \eta\left(r_{r e l}\right)
$$

where $\mathbf{X}$ is the center of mass coordinate $\phi, \theta, \chi$ are the Euler angles connecting the laboratory and a body fixed coordinate system and $r_{r e l}$ stands for the set of relative coordinates. There is nothing inconsistent in specifying half integer values for $j$ in the irreducible rotation matrix elements $\mathcal{D}_{m_{1}, m_{2}}^{j}$ since the rotational group $S O(3)=S U(2) / Z_{2}$ is doubly connected.

This is the point which generalizes for $S U(N)$ : The gauge field transform according to the adjoint representation of the global gauge group, $\mathbf{A} \rightarrow \omega \mathbf{A} \omega^{\dagger}$, c.f. (4.5). The center elements commute with the generators and are represented trivially on the adjoint vectors. The space of global gauge transformation is thus $S U(N) / Z_{N}$. This is a multiply connected space with the fundamental group $Z_{N}$. Thus the wave function which depends on the gauge field may be $\mathrm{N}$-valued. The label of this phase representation of the center is the N-ality, which is the triality 
for QCD. Summarising: the center of the global gauge group is the fundamental group of the global gauge symmetry.

The formal global symmetries of the system may be broken dynamically under certain circumstances. When this happens the charge with respect to the symmetry group ceases to be a good quantum number and the physical states become linear superpositions of components with different charges. The charge of the fundamental group is the multi-valuedness angle, Nality. When the fundamental group symmetry is broken dynamically then the wave functions of the physical states change not only their phase but the absolute magnitude as well under fundamental group transformation.

The last remark about this point is an extension of a technical point. Consider the manybody system (4.11) in a simple connected space. One can always, as was noted, use half-integer $j$ in constructing a wave function. Since $j(j+1)$ is the eigenvalue of the total angular momentum square, $\mathbf{J}^{2}$, and the total angular momentum is the sum of the angular momenta of the particles, $\mathbf{J}=\sum_{a} \mathbf{L}_{a}$, at least one particle must carry half integer orbital angular momentum when $j$ is half-integer. Thus there must be a way to describe half-integer angular momentum states with double-valued wave function in simply connected space. The solution is straightforward and simple. The sufficient condition is that the interaction excludes the particles from certain region in such a manner that the left over of the phase space is multiply connected. Such an interaction can generate multi-valued wave functions even in simply connected space.

After these preliminary remarks we are in the position to give the third interpretation of (4.1). The density matrix for gluons in the presence of an isolated heavy anti-quark is

$$
\rho_{\bar{q}}\left[\mathbf{A}, \mathbf{A}^{\prime}\right]=<\mathbf{A}\left|\mathcal{P}_{\bar{q}} e^{-\beta H}\right| \mathbf{A}^{\prime}>=\int D[\Omega(\mathbf{x})]<\mathbf{A}^{\Omega}\left|e^{-\beta H}\right| \mathbf{A}^{\prime}>\operatorname{tr} \Omega\left(\mathbf{x}_{0}\right)
$$

in the field diagonal representation. The factor $\operatorname{tr} \Omega\left(\mathbf{x}_{0}\right)$ is to guarantee Gauss' law at the location of the test charge. By the help of the invariance property of the group integration, $\int d g f(g)=\int d g f(g h)$, one can easily prove the relation

$$
\rho_{\bar{q}}\left[\mathbf{A}^{z}, \mathbf{A}^{\prime}\right]=e^{-i \frac{2 \pi}{3}} \rho_{\bar{q}}\left[\mathbf{A}, \mathbf{A}^{\prime}\right]
$$

This shows that the gluon wave functional which corresponds to an isolated quark is multivalued. The non-vanishing of (4.1) is just the statement that the gluon states with multi-valued wave functional contribute in the high temperature phase. Had the integration in (4.2) or (4.3) had been performed completely (4.1) would have been vanishing and the states with multi-valued wave functions would have been absent. The appearance of such states in the partition function indicates that the gluons are constrained into a multiply connected region of the configuration space in the high temperature phase and the fundamental group symmetry is broken dynamically.

\section{RIDDLES}

The following arguments are to demonstrate some of the unusual features of the deconfining phase transition:

1. High energy symmetry breaking: The only way to violate a formal symmetry by the dynamics is the spontaneous symmetry breaking. Then the symmetry is broken in the vacuum, at low energy and gets restored at high energy. The center symmetry is broken at high temperature and is restored at low temperature. What is the mechanism of this unusual symmetry breaking pattern ? 
2. Asymptotic states: One may compare the deconfining phase transition with the insulatorconductor Mott transition in solid state physics. In the latter the lattice impurities make the electron wave function localized at high temperature and lead to the breakdown of the conductance. Note that the basic difference between localization and confinement is that while the former allows charged states with zero momentum the latter does not. The thermal averages,

$$
<A>_{t h}=\sum_{n} p\left(E_{n}\right)<n|A| n>
$$

are computed in Quantum Statistical Mechanics by the help of the eigenstates of the hamiltonian, $H\left|n>=E_{n}\right| n>$, and the distribution function, $p(E)$. There are extended electron states on the solid state lattice which provide the main contribution to the partition function in the insulator phase. But there are no finite energy eigenstates of the QCD hamiltonian with net color charge. Where do the deconfined quarks of the high temperature phase come from in QCD?

3. Finite volume effects: According to the second interpretation of the Polyakov line it is the order parameter of a formal symmetry of the partition function and such must be preserved for a finite system. Thus (4.1) is vanishing when the system is placed into a finite volume with open boundary condition in the spatial directions. The first interpretation then assigns infinite energy for the static test charge. Since the phase transition does not changes the ultraviolet properties of the system the divergence in the energy can not be ultraviolet. The finite quantization box provides an infrared cut-off. How can then the energy of a static charge be infinite in a finite box?

\section{SOLUTIONS}

1. High energy symmetry breaking: Though we started with the partition function at finite temperature the situation is formally similar when a general matrix element, (4.8), is considered. Actually, both the partition function and the matrix element show the phase transition for short time in the mean field approximation. To see this first note that the potential energy is negligible compared to the kinetic term for the short time processes. The kinetic energy of the temporal gauge hamiltonian is quadratic in the gauge field and is large if the initial and the final configurations differ for short time. By comparing (4.9) with

$$
-\ln <\mathbf{x}_{f}\left|e^{\frac{\beta}{2} \frac{\partial^{2}}{\partial \mathbf{x}^{2}}}\right| \mathbf{x}_{i}>=\frac{1}{2} \ln (2 \pi \beta)+\frac{\left(\mathbf{x}_{f}-\mathbf{x}_{i}\right)^{2}}{2 \beta}
$$

one sees that $\mathcal{H}[\Omega]$ is minimal for $\Omega(\mathbf{x})=z^{k}$ because this is the case when the initial and the final configurations agree and the static configuration with vanishing kinetic energy dominates the path integral. When the initial and the final configurations differ in the short time limit then the large kinetic energy makes the transition suppressed and $\mathcal{H}[\Omega]$ increases. The maximum of $\mathcal{H}[\Omega]$ is reached in the middle between two center elements, $\Omega=\sqrt{z}$. The free system has no internal scale so the energy barrier between the minima is proportional to $V \beta^{3}$ where $V$ is the three volume. The system is trapped in one of the center minima for short time. Similar periodic structure is found for $\mathcal{H}_{\mathbf{A}_{f}, \mathbf{A}_{i}}[\Omega]$ with minima at $\Omega(\mathbf{x})=z^{k} \tilde{\Omega}(\mathbf{x})$ where $\tilde{\Omega}(\mathbf{x})$ minimizes $-\operatorname{tr} \int d^{3} x\left(\mathbf{A}_{f}^{\Omega}(\mathbf{x})-\mathbf{A}_{i}(\mathbf{x})\right)^{2}$. This simple argument shows that the fundamental symmetry is broken by the kinetic energy for the amplitudes of short time processes. This symmetry breaking mechanism is fundamentally different than the spontaneous symmetry breaking which is 
driven by the potential energy and is effective at low energy, long time. The breakdown of the fundamental group symmetry and the applicability of the mean field solution provides the basis of the parton picture in high energy hadron physics.

2. Asymptotic states: The last equation in (4.1) expresses the fact that the partition function of the sector which comprises that states with a color charge of an anti-quark is nonvanishing. What states contribute to this partition function ? Since the system in question consists of gluons these states belong to the gluonic Fock space. They share an unusual property namely that the total charge seen by local operators in these state is that of an anti-quark. The apparent contradiction that the sum of integers (gluon charge) can not be $\frac{1}{3}$ (quark charge) is circumvented by piling up infinitely many gluons. The limit of an infinite series can be modified by reshuffling the series. An example of a state which has infinitely many plane wave components and belong to the anti-quark charge sector is

$$
\left|\mathbf{A}(\mathbf{x})>_{\bar{q}}=\int d \Omega_{0}\left(\Omega_{0}\right)_{a b}\right| \mathbf{A}^{\Omega_{0}}(\mathbf{x})>,
$$

where $\mathbf{A}(\mathbf{x})$ is an arbitrary localized non-vanishing gluon field configuration, $d \Omega_{0}$ is the invariant integration over global gauge transformations and $\left(\Omega_{0}\right)_{a b}$ is a matrix element of the integral variable. Note that by replacing $\left(\Omega_{0}\right)_{a b}$ by any other matrix element of an irreducible representation of the gauge group the resulting nontrivial state belongs to the given representation. This is the demonstration of the well known fact in Quantum Mechanics that the coordinate eigenstate has non-vanishing projection in all angular momentum subspace. In a similar manner, the gluon state $\mid \mathbf{A}(\mathbf{x})>$ contains components from each charge sector.

It is easy to see that the wave functional of (6.2) is N-valued. Thus the gluonic state surrounding the test quark carries the color charge of an anti-quark and the gauge invariance of (4.1) assures that the dressed test quark is color neutral. The following picture emerges for the deconfining phase transition: At low temperature where the $\Omega(\mathbf{x})$, i.e. end point dependence is weak in the right hand side of (4.8) the $\Omega(\mathbf{x})$ integration is properly performed. This reinforces Gauss' Law and makes the gluonic states neutral in the vacuum sector. At high temperature the infinite dimensional $\Omega(\mathbf{x})$ integration is not carried out properly. The strong end point dependence restricts the integration into a smaller region. This is formally similar to the spontaneous breakdown of symmetries except that it refers now a given matrix element, (4.8), instead of the vacuum. When the projection into the color singlet sector is not implemented properly then states with ill-defined charge show up in the partition function. Since it is the center of the gauge group which breaks down dynamically only the fractional part of the color charge becomes ill-defined. The expectation value (4.1) picks up the contribution of the anti-quark charge components of the gluon states. Thus the deconfining phase transition is the point where a constraint what we impose formally is rejected by the dynamics.

One is attempted to speculate about the following toy-model where a quark flavor, say the top quark, is missing from the electro-weak current. A deep inelastic lepton-hadron collision made on a hadron with non-vanishing top quantum number is interpreted in the framework of this model as the finding of a deconfined quark. Indeed, the top quark which screens the other quark(s) of the hadron is not seen in the experiment. Thus it would have been more appropriate to use the word "screened" rather than "deconfined" for the high temperature phase of the pure gluon system. The quarks can be isolated in this phase only because there are unusual gluon states available which can provide complete screening.

It is worth while noting that the hamilton operator is given as

$$
H_{\bar{q}}=H \mathcal{P}_{\bar{q}}=\int D[\Omega(\mathbf{x})] H \hat{\Omega} \operatorname{tr} \Omega\left(\mathbf{x}_{0}\right)
$$


in the anti-quark sector. This expression is obtained from (4.12) by taking into account $\left[H, \mathcal{P}_{\bar{q}}\right]=$ 0 . The complex phase, $\operatorname{tr} \Omega\left(\mathbf{x}_{0}\right)$, has an effect which is similiar to that of the Wess-Zumino-Witten term in the Skyrme model [9]. It attaches a phase factor to global internal space rotations in such a manner that the hedgehog configurations, the chromomagnetic monopoles in our case, acquire fermi statistics [8]. The gluonic state which screens the deconfined quark can be written as a linear superposition, $\Psi[\mathbf{A}(\mathbf{x})]=\Psi_{0}[\mathbf{A}(\mathbf{x})]+\Psi_{1}[\mathbf{A}(\mathbf{x})]$, where the support of $\Psi_{n}[\mathbf{A}(\mathbf{x})]$ is in the space with even or odd number of monopoles for $n=0$ and $n=1$, respectively. Thus the deconfined quark is such a composite particle of the quark and the screening gluonic cloud that these two components obey boose $(n=1)$ and fermi $(n=0)$ statistics.

An important question hiding in this scenario is whether the unusual gluon states which can screen a quark are stable. The screening cloud around a charge in the electron gas is created by the original charge and disappears in its absence. The strong non-linearity of the gluonic system in particular the instanton solution may make the screening states stable at least semiclassically. If the screening states prove to be stable then the phenomenology of the high temperature gluonic phase should be guided by a simple quark model for these states.

Finite volume effects: This puzzle will be resolved in two steps. The fundamental idea is to explain the vanishing of a quantity like $c e^{-\beta F}$ by the vanishing of the coefficient $c$ rather than using the rather problematical $F=\infty$. We start with the well known two slits thought experiment in Quantum Mechanics. Place a particle source and a detector on the two opposite sides of a wall with two small slits. The location of the detector is chosen in such a manner that the difference of the two paths along which the particles can propagate is a half wave length. The amplitude of finding a particle at the detector is $\psi=c_{1} e^{-i t E_{1} / \hbar}+c_{2} e^{-i t E_{2} / \hbar}$. It is vanishing because $c_{1}=-c_{2}$ and $E_{1}=E_{2}$. How could we give account of the absence of the particles at the location of the detector without knowing about the interference between the allowed paths? The amplitude of finding a particle can generally be written as $\psi=c e^{-i t E / \hbar}$ where $\hbar$ has an infinitesimally small imaginary part to comply causality. In lacking any specific kinematical reason to set $c=0$ we end up arguing for $E=\infty$. But the information that the amplitude is vanishing due to a destructive interference makes it possible to explain the measurement without invoking infinities.

This scenario can be carried over to the internal space of the gluons. The vanishing of (4.1), more precisely (4.3), can be understood in the following manner: Imagine the path integral representation for the matrix element $<\mathbf{A}^{\Omega}\left|e^{-\beta H}\right| \mathbf{A}>$ in the right hand side. We introduce equivalence classes for the configurations $\mathbf{A}(\mathbf{x}, t)$ of the path integral. The configurations $\mathbf{A}(\mathbf{x}, t)$ and $\mathbf{A}^{\prime}(\mathbf{x}, t)$ belong to the same class if their end points can be made equal by a time dependent global gauge transformation, i.e. when

$$
\left\{\begin{array}{l}
\mathbf{A}^{\omega}(\mathbf{x}, 0)=\mathbf{A}^{\prime}(\mathbf{x}, 0) \\
\mathbf{A}^{\omega}(\mathbf{x}, \beta)=\mathbf{A}^{\prime}(\mathbf{x}, \beta)
\end{array}\right.
$$

hold for an appropriate $\omega(\mathbf{x}, t)=\omega(t)$. Each equivalence class can further be divided into $N$ components. This is because $\omega(0)$ and $\omega(\beta)$ must be center elements in order to satisfy (6.4) for $\mathbf{A}=\mathbf{A}^{\prime}$ and $\omega(t)$ and $\omega(t) \omega^{\dagger}(0)$ have the same effect on the gauge field. There is a destructive interference in (4.3) between the components within each equivalence class.

To prove this first note that the gluonic matrix element, $<\mathbf{A}^{\Omega}\left|e^{-\beta H}\right| \mathbf{A}>$, depends on the end points of the trajectories. Since the global center transformation does not change the gauge field this amplitude and the integral measure is the same in each component. The Polyakov line in (4.3), $\Omega\left(\mathbf{x}_{0}\right)$, which takes into account the propagation of the test quark gets multiplied by a 
center element as we move from one component to another. So the sum of the components is proportional to

$$
\sum_{j=1}^{N} e^{i \frac{2 \pi}{N} j}=0
$$

The detector which is prepared to identify an isolated quark remains silent because whenever a quark arrives there it comes through $N$ different paths with destructing interference. The large kinetic energy suppresses $N-1$ paths in the amplitude for short processes and the quarks can be isolated.

\section{DYNAMICS V.S. KINEMATICS}

It is important to distinguish the kinematical constructions from the dynamical assumptions. The only dynamical input to support the arguments above is the non-vanishing of (4.1) for $T>T_{d e c}$. The non-vanishing of an order parameter is always the result of the reduction of the region of an integration, the system being trapped in a part of the whole phase space. How does this happen here? As mentioned in the previous Section the integration in (4.1) is restricted in our case. The formal functional integral is over the space $\mathcal{G}=\prod_{\mathbf{x}} G(\mathbf{x})$ which is the direct product of the gauge group, $G(\mathbf{x})$, at each lattice site. The local symmetries are never broken dynamically so one has to separate off the global gauge group, $G_{0}$, and its center, $C$, by writing

$$
\mathcal{G}=C \otimes G_{0} / C \otimes \mathcal{G} / G_{0}
$$

The integration is not carried out for the first factor in (4.1) above the critical temperature according to the numerical simulations. The second factor is an N-fold connected space which is the source of the $\mathrm{N}$-valuedness of the gluonic wave functional.

A functional integral which is of infinite dimensional can in principle undergo phase transitions. The restricted "symmetry broken" realization of the integral corresponds to a stable dynamical system if the domain of integration is closed under the infinitesimal deformations of the trajectories. This is because the equation of motion and other constraints, such as the Ward identities which express the presence of formal continuous symmetries are written by the help of the canonical coordinate and momentum operator, $\mathbf{A}(\mathbf{x})$, and $\mathbf{E}(\mathbf{x})$, respectively which are multiplicative and derivative operators. The former is diagonal in the coordinate eigenstate basis and the latter generates infinitesimal displacements in the coordinate eigenvalues. Thus the insertion of these operators do not lead out of the domain of integration and consequently can be taken into account properly if the space of trajectories consists of complete homotopy classes.

On the one hand, the configuration space of the gluon system, i.e. the space of field configurations $\mathbf{A}(\mathbf{x})$ is obviously simply connected. But on the other hand, the dynamics may exclude the system from certain region of the configuration space in such a manner that the left over is multiply connected. In our case this amounts to the condition that the internally paralel field configurations, $\mathbf{A}^{(p)}(\mathbf{x})=\mathbf{A} \alpha(\mathbf{x})$, are suppressed by the dynamics in the high temperature phase,

$$
\rho\left[\mathbf{A}^{(p)}, \mathbf{A}^{(p)}\right]=0 .
$$

In fact, (7.2) is needed to make the $N$ components introduced after eq. (6.4) disconnected. The suppression of the long range field is the result of the non-perturbative magnetic screening in the high temperature phase. 


\section{CONCLUSIONS}

Algebraic and topological arguments were presented to demonstrate some unusual features of the high temperature phase of the gluon system. In particular, a new type of dynamical symmetry breaking which is driven by the kinetic energy, a screening mechanism by unusual gluonic states and an explanation of the absence of isolated quarks in the vacuum without evoking infinite energy was given. Though the arguments were exact, i.e. do not involve approximations they remained qualitative only. The detailed quantitative verification of these points with special attention paid to the dynamical quarks is needed in order to fit the phenomena mentioned above into the usual description of the quark-gluon plasma which is based on the partially resumed perturbation expansion.

\section{REFERENCES}

[1] V. N. Gribov, J. Nyiri, Supercritical Charge in Bosonic Vacuum LU-TP-91-15, unpublished.

[2] W. Greiner, B. Müller, J. Rafelski, Quantum Electrodynamics of Strong Fields, SpringerVerlag, Berlin, 1981.

[3] M. Oleszczuk, J. Polonyi, Canonical versus Grand Canonical Ensembles in QCD, submitted to Z. Phys.; M. Faber, O. Borisenko, G, Zinovjev, Triality in QCD at Zero and Finite Temperature: a New Direction, to appear in Nucl. Phys. .

[4] S. Mandelstam, Phys. Rep. 23C 245 1976; G. t' Hooft, Nucl. Phys. B190 455 1981; H. B. Nielsen, P. Olesen, Nucl. Phys. B61 451976.

[5] K. Johnson, L. Lellouch, J. Polonyi, Nucl. Phys. B367 675, 1991.

[6] L. Susskind, Phys. Rev. D20 2610 1979; A. M. Polyakov, Phys. Lett. 72B 4471978.

[7] J. Polonyi, Zs. Schram, Nucl. Phys. Proc. Suppl. B42 9261995.

[8] J. Polonyi, in Quark-Gluon Plasma, edt. R. Hwa, World Scientific, 1988.

[9] E. Witten, Nucl. Phys. B223 422 1983, ibid 4331983. 